\section{RevistadePolírica Económica y Desarrollo Sostenible}

Centro Internacional de Política Económica para el Desarrollo Sostenible
Revista de Política Económica y Desarrollo Sostenible

EISSN: 2215-4167 • Vol. 3 (1) • Enero- Junio, 2018: 44-63

DOI: http://dx.doi.org/10.15359/peds.3-1.3

URL: http://www.revistas.una.ac.cr/politicaeconomica

Revista electrónica semestral publicada por el Centro Internacional de Política Económica para el Desarrollo Sostenible

Universidad Nacional, Lagunilla, Heredia Apartado 2393-3000

\title{
Desarrollo territorial de Guanacaste desde el enfoque de sistemas territoriales de producción agroalimentaria
}

\author{
Guanacaste's Territorial development since the territorial systems approach of \\ agri-food production
}

\author{
Stephanie Porras Acevedo ${ }^{1}$ \\ ORCID: https://orcid.org/0000-0001-7269-5794
}

steph5pent@gmail.com

Fecha de recibido: 26 de junio del 2017- Fecha de corrección: 15 de enero del 2018 - Fecha de aceptación: 28

de enero del 2018

\section{Resumen}

El objetivo de este artículo es identificar los factores territoriales que impulsan o frenan el establecimiento de un sistema territorial de producción agroalimentaria (STPA), bajo el enfoque de desarrollo territorial, en el cantón de Carrillo de la provincia de Guanacaste, Costa Rica. Debido al dinamismo que ha experimentado la provincia en los últimos años y al desplazamiento de la actividad agrícolas desde los 90, debido a la actividad terciaria, se busca investigar aquellos factores que promuevan el crecimiento considerando las condiciones que proyectos actuales, como la creación del Mercado Regional PIMA en Carrillo, la ampliación terminal de carga en el aeropuerto Daniel Oduber Quirós en Liberia y la construcción del canal seco de La Cruz hacia Puerto Limón pueden ofrecer. Como resultado del análisis se proponen los componentes de un sistema territorial de producción agroalimentaria para la provincia, considerando las características de las empresas y la sinergia que estos actores deben tener con las instituciones. El artículo concluye que los STPA promueven una alta asociatividad y encadenamientos entre empresas, gobierno y recursos disponibles. Además, incluye el medio ambiental como una variable a circunscribir en los procesos. En su conjunto, los STPA pueden considerarse una alternativa para promover la seguridad y soberanía alimentaria de las regiones, a través del abastecimiento de un mercado con productos locales.

Palabras clave: Factor de producción, territorio, desarrollo, producción alimentaria, seguridad alimentaria.

\section{Abstract}

The purpose of this article is identify the territorial factors that can drive or hinder the establishment of a territorial system of agri-food production (TSAP), under the territorial development approach in the canton of Carrillo, Guanacaste, Costa Rica. Since 1990, Guanacaste has experienced a displacement of agricultural activity

1 Máster en Gerencia del comercio Internacional por el Centro Internacional de Política Económica para el Desarrollo Sostenible, Universidad Nacional. Licenciada en Economía por la Escuela de Economía de la Universidad Nacional. Actualmente se desempeña como docente universitaria en la Universidad de Costa Rica y la Universidad Nacional, Sede Liberia. Es socia consultora de la empresa Asesoría y Consultorías Porras \& Vásquez 
Revista de Política Económica • EISSN: 2215-4167

Vol. 3 (1) • Enero- Junio, 2018: 44-63

DOI: http://dx.doi.org/10.15359/peds.3-1.3

Porras Acevedo

URL: http://www.revistas.una.ac.cr/politicaeconomica

due to tertiary activity. In this article, we investigate factors that can promote growth considering the current conditions that regional new projects offer, for example: the creation of the PIMA Regional Market in Carrillo, the cargo terminal extension at the Daniel Oduber Quirós airport in Liberia and the construction of the dry channel from La Cruz to Puerto Limón. As a result of the analysis, the components of a territorial agri-food production system for the province are proposed, considering the characteristics of the companies and the synergy that these bands should have with the institutions. The article concludes that TSAP promote a high associativity and linkages between companies, government and available resources. In addition, it includes the environment as a variable to considerer in this processes. As a whole, this kind of system can be consider as an alternative to promote the food security and sovereignty of the regions, through the supply of a market with local products.

Keywords: Production factor, territory, development, food production, food security.

\section{Introducción}

La provincia de Guanacaste representa un 20\% del territorio costarricense. Posee una extensión de 10.140.71 kilómetros cuadrados y cuenta con 359,020 mil habitantes, según información del Instituto Nacional de Estadísticas y Censo (INEC) para el año 2014. El cantón más habitado es el de Liberia, seguido por Santa Cruz, Nicoya, Carrillo y en menor porcentaje La Cruz. El clima se caracteriza por ser tropical seco con dos estaciones bien definidas, sin embargo, en los últimos años se ha visto afectada por el Fenómeno El Niño, provocando temporadas secas muy extensas, disminuyendo las fuentes de agua y dificultado la producción agroalimentaria y el turismo. Según el Instituto Meteorológico Nacional (2015), la tendencia del 2014 se repitió en el 2015, ocasionando que la época lluviosa se acortará de seis a dos meses.

La llamada "Pampa Guanacasteca”, según el Instituto Nacional de Estadísticas y Censo en su Encuesta Nacional de Hogares (ENAHO, 2014), se ha caracterizado por ser dadora de recursos para el país, produce el 35\% de energía eléctrica ya sea de fuente hidroeléctrica, geotérmica y solar. Además, señala el rezago que experimenta la provincia pese a todos los recursos que posee, en infraestructura vial solo el 50\% de sus carreteras se encuentran con lastre y se caracteriza por ser una de las regiones más pobres del país con un 33.2\% para el 2014; distribuidos entre pobreza no extrema y pobreza extrema.

De acuerdo con datos del Comité Sectorial Agropecuario de la Región Chorotega (2007), a partir de los años noventa, se presenta a Guanacaste con un rostro turístico, 56\% de la inversión se tornó a este sector, cuando antes el predominio era el sector primario. En 1963, el 73\% de la Población Económicamente Activa (PEA) se dedicaba a la actividad primaria, mientras que para el 2009 paso a ser un $18 \%$ versus un $67 \%$ dedicado a la actividad terciaria. Esto claramente se debe a los grandes proyectos turísticos desarrollados en estos últimos veinte años.

Pese a los encadenamientos productivos que se puedan generar entre ambos sectores, primario y terciario, esto no se logra en la provincia dado que el desarrollo de la actividad ha disminuido y la diversificación de los productos se ha limitado principalmente a: arroz, azúcar, 
naranja, melón, maíz, frijol, con mayor número de hectáreas dedicadas al cultivo (Comité Sectorial Agropecuario de la Región Chorotega, 2007).

Durante el año 2014, en las exportaciones provenientes de la región Chorotega destaca la importancia del sector agropecuario, en conjunto con los sectores agrícola, alimentario, pecuario y pesca, que juntos contribuyen con el $82,5 \%$ de las exportaciones de la región, siendo Liberia el que cuenta con mayor presencia de fincas (14.9\%), seguido por Santa Cruz (12.8\%), Nicoya (11.8\%), Cañas (10.4\%), y Carrillo (5.7\%) (Secretaría Ejecutiva de Planificación Sectorial Agropecuaria, 2014)

Con este contexto, el propósito del artículo es caracterizar los elementos potenciales del Cantón de Carrillo para el establecimiento de un Sistema Territorial de Producción Agroalimentario (STPA), considerando los programas actuales direccionados por el Gobierno de Costa Rica como lo son la construcción del Mercado Regional Chorotega (PIMA) y la ampliación de la terminal de carga del aeropuerto Daniel Oduber. Sin embargo, debido a las limitantes de información existentes en lo referente a la oferta de producción del cantón se toman los datos generales de la provincia de Guanacaste, con el fin de identificar las características deseables para la conformación del sistema.

\section{Metodología}

Para la caracterización de los elementos potenciales del Cantón de Carrillo que aportarían en el establecimiento de un Sistema Territorial de Producción Agroalimentario (STPA), se identificaron los siguientes grupos de interés:

1. Los mediadores: Ministerio de Agricultura y Ganadería, Programa Integral de Mercado Agropecuario, ADC \& HAS: CORICORP, PROCOMER, Municipalidad de Carrillo, Cámara Nacional de Agroindustria y Agricultura.

2. Actores identificados como oferentes de productos agroalimentarios, divididos a su vez en tres grupos: a) Se reconocieron a un total de 816 productores en el cantón de Carrillo distribuidos de la siguiente manera: 389 ganadería, 209 caña azúcar, 166 maíz, 29 frijol y 23 Arroz. b) Las asociaciones y cooperativas del cantón de Carrillo, que en total sumaron 11, entre las que se encuentran: Asociación Hortícola San Blas, Asentamiento la Hurraca, La Piragua, Asociación Grupo de Mujeres Emprendedoras de Corralillos GRUMEC, Asociación Cámara de Ganaderos de Carrillo, Cooperativa Agropecuaria y de Servicios Múltiples, Asociación de productores agropecuarios Luchadores Rio Cañas Nuevo, Asociación de mejoramiento de vida de Playas del Coco. En esta lista se incluyeron las cámaras de ganaderos para valorar proyectos de diversificación al aplicar el cuestionario.

3. Cooperativas y empresas destacadas en la provincia que realizan exportación ya sea directa o indirectamente.

Para cada grupo se designó una metodología de trabajo que permitiera el logro de los 
objetivos propuestos. Ello se cumple por medio de entrevistas estructuradas a los participantes del primer grupo, análisis base de datos de 816 productores del cantón y aplicación de cuestionario a las 11 asociaciones y cooperativas del cantón de Carrillo y por último entrevistas estructuradas a 9 de las principales empresas exportadoras de la provincia.

\section{Marco conceptual}

\subsection{Desarrollo y sistemas territoriales de producción agroalimentaria}

Existe un alto grado de relación en los términos crecimiento y desarrollo y aunque ambos son estrechamente cercanos, sus alcances tienden a generar confusión. El primero es de carácter cuantitativo, mientras que el segundo se puede ligar a aspectos cualitativos, es decir, interesa más la calidad que la cantidad.

Son muchas las teorías que definen el concepto de desarrollo, por ejemplo, la teoría de desarrollo local (Alburquerque, 2003) busca el análisis de las potencialidades endógenas de factores económicos y no económicos para una comunidad en específico; mientras que la de desarrollo territorial (Alburquerque, 2006), aporta a incorporar el marco institucional, el cual debe crear condiciones favorables que faciliten la incorporación de innovaciones y nuevos emprendimientos.

En esta última, las instituciones no se refieren únicamente a organizaciones, sino a redes, normas y reglas explicitas o implícitas del comportamiento humano en un territorio, tal y como se explica a continuación:

El enfoque del desarrollo territorial supone un planteamiento integral y contextualizado sobre el funcionamiento de las economías. Dicho enfoque conduce a una estrategia que debe acompañar los esfuerzos de carácter macroeconómico y la promoción de exportaciones con actuaciones orientadas a lograr la introducción de innovaciones tecnológicas, de gestión y socio-institucionales en el conjunto de los diferentes sistemas productivos locales y tejidos de empresas existentes (Alburquerque, 2006, p. 5).

Estas regiones o localidades deben planificarse con el fin de crear un sistema genérico, donde el crecimiento y desarrollo interactúen en beneficio de la localidad, como un todo, es decir, interesa identificar los elementos cualitativos que permitan la identificación de los factores territoriales que asentirían el establecimiento de sistemas territoriales de producción agropecuaria dirigido a medianos empresarios.

Además, se debe entender que los sistemas territoriales vinculan el aprendizaje y la innovación dentro de sus procesos en un territorio determinado. Al respecto y siguiendo a Gallego (2009), la interacción de las empresas en estos territorios dependerá de estas variables. 
La teoría de desarrollo territorial parte del reconocimiento de factores territoriales que definen a los territorios innovadores, no así el estudio de las empresas como dinamizadores del desarrollo en determinado espacio visto desde el enfoque de Clúster desarrollado por Michael Porter. Autores como Benko y Pecqueur (2001) y Colletis y Pecqueur (1995) y Méndez (2002), realizan la distinción de estos factores como recurso y activos, definiendo que:

- Recursos disponibles en determinado territorio, sean estos recursos naturales, población ocupada, ahorro disponible, ingreso disponible, inversión, infraestructura, entre otros.

- Recursos y activos específicos: mano de obra calificada, experiencia laboral, servicio de apoyo y formación, marco social e institucional, entre otros.

Para fines del estudio, se entiende recursos disponibles como aquellos con los que cuenta la provincia a nivel macro, por ejemplo: hectáreas de producción, mientras que los recursos y activos específicos se entienden desde una perspectiva micro, por ejemplo hectáreas de producción según producto.

Siguiendo a Méndez (2002), los elementos a considerar para el desarrollo territorial integrado se muestran a continuación:

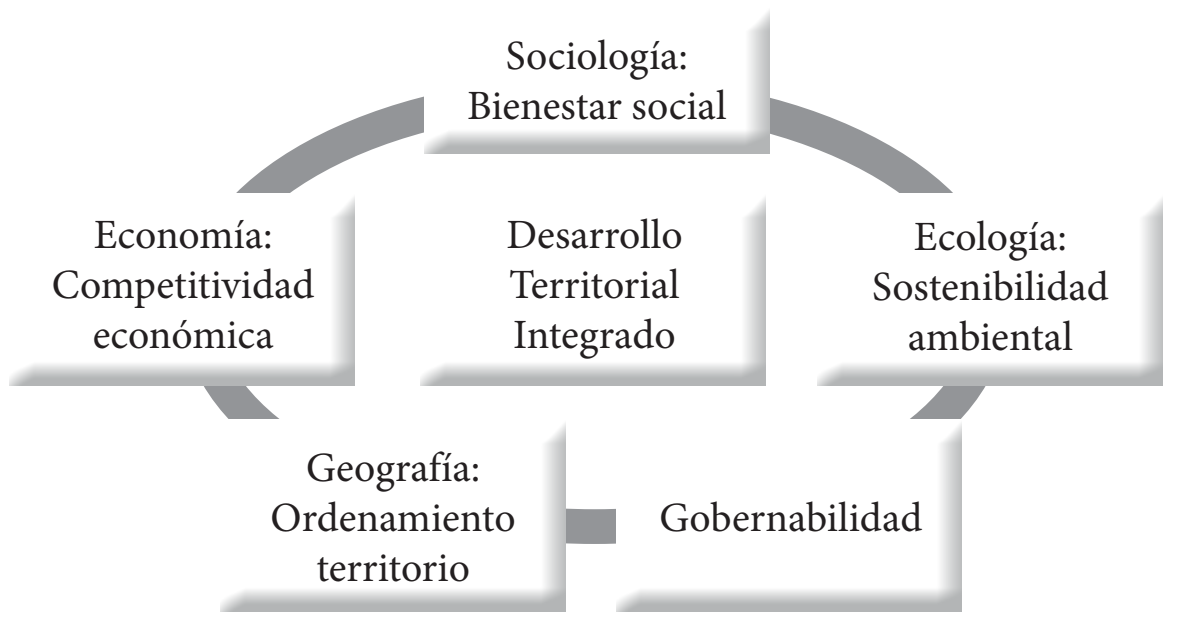

Figura 1. Principales elementos integrantes del concepto de desarrollo territorial integrado.

Fuente: Méndez, 2002

El desarrollo territorial corresponde a toda una evolución del concepto para lograr integrar los recursos potenciales de una zona como parte de los agentes dinamizadores de este; un tema poco abordado desde la óptica de la competitividad. En el presente artículo se adopta el concepto de desarrollo territorial desde la perspectiva de Méndez, por lo que se busca identificar a actores sociales, los cuales determinan a los oferentes y demandantes, las empresas, el entorno 
económico, el ambiente y la Gobernabilidad; la cual se entiende a partir de las instituciones que se interrelacionan, tal como indica la ilustración.

\section{Resultados}

En este apartado se desarrollan los componentes territoriales que determinan las principales características tanto del cantón de Carrillo y como de la provincia de Guanacaste. El punto de partida es reconocer que existe un contexto institucional y un marco jurídico propio de la legislación costarricense que debe considerarse para lograr la integración de un sistema territorial. Para efectos del estudio dirigido al sector agroalimentario, se ha incluido instituciones tales como: Ministerio de Agricultura y Ganadería, Programa Integral de Mercado Agropecuario, ADC \& HAS: CORICORP, PROCOMER, Municipalidad de Carrillo y Cámara Nacional de Agroindustria y Agricultura.

A su vez, es necesario resaltar que los hallazgos se encuentran generalizados a la provincia y al estilo de desarrollo que ha predominado; esto debido a que en el cantón de Carrillo las asociaciones y cooperativas no presentaron un mayor crecimiento y el principal producto cultivado correspondía a la caña de azúcar, el cual fue excluido del estudio, por la imposibilidad de rotación de cultivo en los suelos dedicados a esta actividad.

Los resultados se organizan abordando en primer lugar el inventario de recursos disponibles y en segundo lugar los activos específicos.

\subsection{Inventario de recursos disponibles}

En relación con las actividades productivas de la provincia, existe un claro desplazamiento del sector primario por el sector terciario, en particular por el turismo y su extensa gama de servicios. A este aspecto se le adhieren los proveedores de insumo y servicios, así como la dotación de maquinaria y equipos que requieren las empresas para operar su actividad productiva, logrando así el trade off entre los sectores, los cuales no son interdependientes.

Costa Rica ha experimentado grandes cambios a nivel agrícola: pasó de ser un país agroexportador a un modelo de sustitución de importaciones y posteriormente a la promoción de las exportaciones a partir de la década de 1990. El desplazamiento de la actividad primaria se ha generado por el turismo el comercio de bienes y servicios. Mientras que la oferta de productos ha logrado la diversificación ofreciendo una gama mayor de estos, lo cual favorece el abastecimiento interno de la provincia.

Por su parte, dentro de las instituciones involucradas, algunas han sufrido modificaciones, como fue el caso del Instituto de Desarrollo Agrario (IDA), que pasó a ser el Instituto de Desarrollo Rural (INDER). De igual forma, se nota la participación de nuevas instituciones gubernamentales que lideran el desarrollo de la provincia, como el Instituto Costarricense de Turismo (ICT), el cual gana protagonismo a partir de 1991 y hasta la actualidad. 
En Guanacaste, el desplazamiento de las actividades productivas y la organización territorial se ven direccionadas por políticas públicas de incentivos a los diferentes sectores. Antes de 1990 se tiene el abandono del estado paternalista a uno que busca la sustitución de importaciones (Sánchez, 2016), por lo cual se busca una reestructuración del ordenamiento territorial y una readecuación parcelaria.

Después de 1991 y hasta la actualidad, debido a esta readecuación parcelaria, se logra la diversificación productiva. Los productos tradicionales como los granos básicos pierden participación, pero se busca la producción de bienes atractivos al mercado externo, el cual se ha dinamizado. Los productores ya no compiten en el mercado interno, sino que deben buscar técnicas para lograr colocar sus productos en el mercado internacional.

Para el periodo anterior a 1990 la provincia de Guanacaste poseía una infraestructura básica. La demanda de vías o caminos de primera no era la prioridad, ya que la industria azucarera era la que mayor exportaba y lo realizaba por vía marítima, por lo cual los gobiernos no incentivaban esta mejora.

En lo que respecta al recurso humano, se ha visto limitado a la escasa demanda de empleo. Según el INEC (2014), Guanacaste es una de las provincias con mayor tasa de desempleo en la actualidad. Pese a que la diversificación de la producción agrícola ha permitido el asentamiento de nuevas empresas, esta no ha logrado absorber, en conjunto con el sector segundario y terciario, la totalidad empleada.

Sobre los recursos naturales, estos deben de resguardarse y se debe prestar especial atención a la dotación de factores que posee la provincia e instaurar medidas de control que promuevan culturas eficientes y sustentables, para que la explotación de los recursos no exceda la tasa de extracción de estos.

En cuanto a la inversión, esta se realiza o transfiere mediante proyectos liderados por el gobierno central y las principales instituciones líderes del desarrollo de la provincia. Esto entendiendo inversión como pública y no privada.

\subsection{Recursos y activos específicos}

En esta sección se presenta un detalle de los recursos y activos con los cuales cuenta la provincia, en relación con la oferta disponible (producción), la cadena de suministros (distribución), el acceso a mercados (innovación y certificaciones requeridas), la gestión empresarial (mano de obra calificada, experiencia laboral, servicio de apoyo y formación) y el marco social e institucional.

\subsubsection{Descripción de la oferta disponible}

Se han conformado tres grupos para el estudio de las características de los recursos y activos 
Revista de Política Económica • EISSN: 2215-4167

Vol. 3 (1) • Enero- Junio, 2018: 44-63

DOI: http://dx.doi.org/10.15359/peds.3-1.3

Porras Acevedo

URL: http://www.revistas.una.ac.cr/politicaeconomica

específicos. El primero está conformado por las instituciones, el segundo por las asociaciones y cooperativas del Cantón de Carrillo y el tercero por cooperativas y empresas exportadoras a nivel de la provincia.

\subsubsection{Asociaciones y cooperativas de Carrillo}

Para este estudio se logró contar con siete grupos identificados, de ellos hay cuatro con modelo de asociación, uno de cooperativa y dos que corresponden a asentamientos. Todos, en promedio, cuentan con 21 asociados y 94 hectáreas. Los productos destacados corresponden a mango, caña y pipían. Destaca la Asociación GRUMEC, la cual, con una hectárea, logra producir mediante hidroponía una gran variedad de productos.

En cuanto al nivel de ingresos facturado para el 2015, tienen un promedio de ingreso mensual de \$2.083.333, es decir, cada asociado recibe un ingreso promedio mensual de $\$ 118.623,00$. Otras características de estas empresas es que no se encuentran exportando, no han exportado de forma directa o indirecta. Sin embargo, cuando se consulta si estarían dispuestos a realizar alianzas con empresas para colocar sus productos indican que sí las realizarían, aportando las siguientes razones de mayor peso: forma segura de colocar producción, ingresos asegurados y facilidad de recursos.

En lo referente al origen de los insumos para la producción, las empresas realizan las compras a proveedores cercanos a su cantón principalmente, debido a que no las encuentran en sus inmediaciones de operación, ya que no se encuentran proveedores que satisfagan sus requerimientos, como si los encuentran en otras localidades de Guanacaste y en el país. Entre los proveedores destaca El Colono, Agroveterinaria Liberia y un proveedor de San Carlos, Inversiones Estrella Mar Azul.

Debido a su estructura, estas asociaciones y cooperativa no cuentan con certificaciones, ni con un departamento de investigación y desarrollo. Sin embargo, cuando se les consultó si han realizado innovaciones, contestan positivamente y atribuyen estas innovaciones principalmente a acciones como inclusión de nuevas especies, producción hidropónica y mejora en procesos, acompañamiento con el INDER y el Instituto Nacional para el Fomento Cooperativo (INFOCOP).

Otro aspecto es el destino que tiene la producción, la cual se vende, como era de esperarse, en el mismo distrito en mayor cuantía y, en menor volumen, en otras zonas del país. Las fuentes de financiamiento de estas empresas son del Estado, principalmente, y de reinversión de utilidades. En su mayoría, los productores han obtenido las parcelas por donación, por lo cual cabe destacar que estos necesitan continuidad y asesoría para que se vuelvan exitosos y no se mantengan con producciones de supervivencia, como lo hacen actualmente.

Se ha realizado un acercamiento por parte del Programa Integral del Mercado Agropecuario, con su acrónimo PIMA para promover el proyecto, sin embargo, las opiniones se encuentran direccionadas a no estar preparados y no poder competir con grandes empresas a las que se les pueda 
facilitar cumplir las exigencias presentadas por el PIMA. Bajo esta línea, las empresas muestran el deseo de las asociaciones y la cooperativa de participar, no obstante, reconocen sus debilidades y la necesidad de establecer alianzas o incluir nuevos productos para garantizar la participación.

Referente a los STPA, las empresas no conocen el término, pero han escuchado de la conformación de clúster para la integración de proveedores de la zona con el sector hotelero que se desarrolla en el cantón de Carrillo.

\subsubsection{Cooperativas exportadoras}

Estas cooperativas poseen características similares. Tres de ellas poseen su plan de planeamiento estratégico elaborado por INFOCOP. También realizan exportación indirecta por medio del Consorcio de Cooperativas Cafetaleras de Guanacaste y Montes de Oro (COOCAFE), creada en 1988 para procesar y comercializar el café de sus afiliados.

Asimismo, con un promedio de 51 años en el mercado, estas tres cooperativas (Coopecerroazul, Coopepilangosta y Coopeldos RL) han brindado trabajo e ingresos a las familias de los asociados, facturando una suma mayor a los $\$ 51,000,000.00$ millones de colones.

\subsubsection{Empresas exportadoras}

Estas empresas poseen características en común en la producción de melón. El promedio de años operando es de 27 años, excluyendo a JM Ivando que tiene solo 9 años en el mercado.

En cuanto a hectáreas cultivadas, la que posee mayor extensión es Exporpack, seguida por Melones de la Península y Costeña, con más de 200 hectáreas. Además, son empresas que facturan anualmente más de $\$ 51,000,000.00$.

Existe un predominio de medianas empresas en la zona, por lo que el perfil de las contrataciones corresponde a colaboradores del área administrativa y gerencial. Cabe destacar que existe un elemento temporal en la contratación del personal, ya que para la temporada del melón la contratación de personal asciende a 150 personas en promedio, pero cuando terminan la temporada las planillas disminuyen de 50 a 25 colaboradores.

\subsubsection{Cadenas de suministros}

La administración de la cadena de suministros encierra oportunidades para mejorar el costo o el servicio al cliente, permitiendo la coordinación y la colaboración entre los miembros de los canales de flujos. De esta manera, conviene determinar los encadenamientos que generan estas empresas a la región y el origen de los insumos utilizados. De estos, un $61 \%$ proviene del territorio nacional, mientras que solamente el $22 \%$ es comprado en el extranjero. Se da un menor porcentaje de compra en el mismo distrito, lo cual se relaciona al hecho de que las empresas se localizan en poblados poco desarrollados. 
Otro proceso que caracteriza las cadenas de suministros es el papel de las certificaciones, las cuales incorporan una serie de parámetros que impulsan a las empresas a tener proveedores con comportamientos similares en cuanto a las buenas prácticas productivas y sociales.

Las empresas en estudio incorporan 14 certificaciones en sus estructuras productivas. De estas, tres son nacionales, como Esencial Costa Rica, Carbono Neutro y Buenas prácticas agrícolas; dos de segunda parte (solicitas por clientes), como Starbucks, Tesco y Fyffes; mientras que las restantes son para acceder a mercados como Global Gap, Supply Chain Security y Rain Forest Alliance. Costeña S. A. y Exporpack S. A. son las que mayor número de certificaciones poseen. Cabe destacar el papel de Costeña S. A., la cual posee un menor número de hectáreas en comparación a la segunda.

La innovación dentro de las empresas es constante, muchas asocian estos procesos a las certificaciones y a las exigencias que se les imponen en los requisitos de cumplimiento. El $87 \%$ de las empresas incorporaron este proceso para el periodo 2015, destacando innovaciones principalmente en productos, creando nuevas variedades, nuevos productos que sean más amigables con el ambiente, para producir frutas de mejor calidad, en segundo lugar se encuentra la mejora en procesos relacionadas con las formas de trabajo como la preparación del suelo, industrialización de la producción (en el caso de Coopeldos), preparación del café por medio de injertos.

En cuanto a organización, se presentaron cambios a nivel de razón social, compras de sistema de información para estadísticas y adecuación administrativa. Por último, el rubro otros corresponde a la construcción del laboratorio para investigación y desarrollo por parte de Melones de la Península. Esta última iniciativa conduce a la caracterización siguiente referente a la incorporación de un departamento de investigación y desarrollo. De las ocho empresas, tres de ellas poseen este departamento: Exporpack (1997), Costeña (2015) y Melones de la Península (2015).

\subsubsection{Acceso a mercados}

En cuanto al acceso a mercados, se hace referencia a la colocación de los productos, sean estos nacionales o extranjeros. A nivel externo, se identifican los principales destinos, los cuales se relacionan directamente con las certificaciones adquiridas. Por otra parte, se realiza la caracterización de los medios por los cuales estas empresas exportan y la utilización que realizarían a la ampliación de la terminal de carga del aeropuerto Daniel Oduber, en Liberia.

La distribución de la producción entre el mercado local y el internacional oscila entre el $60 \%$ y el $100 \%$ en el mercado internacional para todas las empresas y cooperativas. Solamente la empresa JM Ivando coloca el $100 \%$ de su producción a nivel externo, mientras que la que mayor porcentaje a nivel nacional coloca corresponde a Coopeldos. Exporpack coloca el 9\% de la producción en el mercado local, la cual corresponde a melón de segunda que no pasa los estándares internacionales y por lo tanto se vende a comercios de la zona o la agroindustria para su industrialización en un producto como la venta de fruta picada. 
Estas exportaciones se realizan principalmente por vía marítima; sin embargo, como el puerto de salida se ubica en la provincia de Limón, es necesario de igual forma llevarlo por vía terrestre. Este traslado de la fábrica al proveedor puede durar de 15 a 22 días según el destino. El principal medio de exportación es el terrestre, esto debido a que las cooperativas trasladan parte de la producción a COOCAFE y de ahí esta lo lleva a los destinos de exportación. El 6 \% exportado por vía aérea corresponde a la exportación realizada por Melones del Sol a Canadá en el 2015.

Es importante destacar que todas las empresas y cooperativas entrevistadas indican que realizarían alianzas para exportar mayor volumen. En caso de Melones de Sol, parte de la producción que venden en el mercado local es para Exporpack, que posteriormente lo exporta a sus principales mercados de destino.

Aunado a esto, el $50 \%$ indica que utilizaría la terminal de carga y el otro $50 \%$ indica que no, ya que es demasiado costoso para el volumen de exportación que tienen; sin embargo, consideran que deben evaluar costos de transporte y determinar el INCOTERM a negociar, ya que podría reducirse el tiempo de traslado al proveedor.

Referente a los mercados extranjeros de destino, llama la atención JM Ivando, quienes colocan el $100 \%$ de su producción en tres destinos (Holanda, Inglaterra e Italia) gracias a las certificaciones Global Gap y Rain Forest Alliance. El país al cual mayor número de empresas exportan es EE. UU., mientras que el mercado europeo se encuentra fragmentado en varios países de destino.

\subsubsection{Gestión empresarial}

Para este rubro es importante rescatar nuevamente la contribución de las certificaciones en la elaboración de la gestión empresarial de la población entrevistada, esto debido a que parte de los elementos de evaluación se encuentran direccionados a crear sistemas de gestión eficientes, desde la administración de la empresa, hasta los procesos operativos llevados a cabo en el campo.

Las empresas señalan como principales fortalezas la calidad del producto atribuible a las certificaciones, la infraestructura y los activos fijos, la estabilidad empresarial, la experiencia internacional, la asociatividad (COOCAFE, ICAFE), el excelente ambiente laboral y el personal capacitado. Mientras que sus debilidades son muy variadas y con necesidad de inversión para suplirlas. Adicionalmente, se agrega la falta de mano de obra a nivel local, la cual por lo general es nicaragüense en temporada de cosecha. La disponibilidad de tierras es un componente que se relaciona con la dotación de factores, en la cual la tierra es un factor fijo.

Las fuentes de financiamiento identificadas muestran la solidez de la empresa. La reinvención de las utilidades y el crédito de proveedores, más el adelanto, señalan que la empresa está al tanto manejar los tiempos de recuperación y rotación de capital. Por otra parte, la fuente de financiamiento por parte de entes gubernamentales se presenta en mayor cuantía en las cooperativas. 
Revista de Política Económica • EISSN: 2215-4167

Vol. 3 (1) • Enero- Junio, 2018: 44-63

DOI: http://dx.doi.org/10.15359/peds.3-1.3

Porras Acevedo

URL: http://www.revistas.una.ac.cr/politicaeconomica

\subsubsection{Participación en mercado regional}

Para la búsqueda de los oferentes se han realizado reuniones de acercamiento con los productores. La importancia del mercado regional en el estudio es que se ubicará en el distrito de Sardinal de Carrillo, lo cual constituirá en una fuente de empleo directo y la oportunidad de que productores locales coloquen sus productos a una demanda de altos estándares que actualmente se abastece de productores de zonas externas a la provincia.

Pese a que las empresas y las cooperativas encuestadas no conocen sobre la conformación de mercados regionales, sí conocen sobre el proyecto PIMA como tal y se encuentran investigando para participar en él. Se han creado algunas disconformidades con respecto a los estudios realizados para determinar los oferentes, ya que estas empresas y cooperativas consideran que en su mayoría el proveedor local no podrá abastecer el mercado.

La diversificación es el principal reto que las empresas tendrían, por lo cual implementar alianzas o asociatividad les traería beneficios en el corto plazo para garantizar la participación. El $63 \%$ de las empresas considera incluir nuevos productos con el fin de lograr esta participación en el PIMA. Entre los productos que indican que podrían ampliar dentro de su oferta por poseer tierras aptas y el conocimiento sobre la rotación del cultivo se encuentran: maracuyá, granada, chile dulce, chile jalapeño, tomate, elote, frijol tierno, sandía, pipián, pitaya, hortalizas y legumbres.

Por último, las empresas y cooperativas indican que la conformación de alianzas y asociatividad estaría incentivada principalmente por tener una mayor oferta de productos, es decir, concursar por un estar asociado al volumen de producto ofertado; seguido por la disminución de costos y en última instancia la reducción de la curva de aprendizaje del cultivo de un nuevo producto.

\section{Conocimiento de las empresas de los STPA}

El $62 \%$ de empresas y cooperativas entrevistadas conocen qué es un STPA, mientras que el 38\% restante no lo conocía. Sin embargo, al mencionar la definición y terminología similar, se ubican en el contexto y señalan que es importante el incentivo de un STPA, ya que lo que le está haciendo falta al sector agroalimentario es esa posibilidad de integración y vinculación con todos los sectores.

Todas las empresas consultadas coinciden en que debe implementarse esta sinergia, para lograr incentivar el sector primario y destacan las siguientes características como ventajosas para lograrlo:

- La preocupación del entorno ambiental es importante ya que si no se preservan los recursos con lo que se dispone actualmente, no se contará con estos en el largo plazo. El abastecimiento de agua, por ejemplo, está creando conciencia en las empresas, por lo que este es uno de los elementos a incorporar de manera inicial en las tareas para los STPA.

- La diversificación es otra variable a atender en el corto plazo, ya que actualmente la oferta es de dos a tres productos por empresa y cooperativa en estudio, los cuales son productos 
no tradicionales y de alto valor en el mercado internacional, pero esto no asegura la alimentación de una población que consume granos básicos como parte de su dieta.

- La necesidad de capacitación representa ese acercamiento de las instituciones a las empresas. No solo basta con financiar proyectos, sino también es necesario brindar seguimiento hasta que las empresas sean eficientes y puedan administrarse de manera independiente.

- La ampliación de condiciones del mercado y las estrategias de desarrollo son dos elementos que van de la mano: al apoyar el sector agropecuario como una estrategia de desarrollo, no solo se asegura la producción de la zona sino que también se incentiva espacios para colocar el producto que no se producía por falta de un mercado demandante.

De esta manera, se concluye con los principales componentes y características que deben poseer las empresas para participar en un STPA, el cual tiene como principal herramienta la conformación de un mercado regional, en el cual las empresas deben de diversificar su producción y buscar medidas de innovación que promuevan la preservación de los recursos con procesos de producción eficiente.

\section{Propuesta de un STPA en Guanacaste}

La propuesta de este STPA como una alternativa de desarrollo del cantón de Carrillo va dirigida a aprovechar los recursos con los que dispone el cantón y generalizarlos a la provincia de Guanacaste, con el fin de identificar a los productores locales equiparando sus debilidades y potenciando sus fortalezas, para determinar los puntos de mejora y las necesidades.

El aprovechamiento de proyectos y recursos es otro tema, el cantón y los productores locales son capaces de producir gran cantidad de los bienes que se demandan a nivel local, por lo cual deben de buscar alternativas para participar en el Mercado Regional y competir con productores de otras zonas del país que buscarán colocar sus productos en la región.

Se ha realizado la propuesta de tres "escenarios desafío", con el fin de determinar los beneficios que conllevará al cantón de Carrillo el establecimiento de un STPA. Para ello se identificaron dos grupos de variables. El primero está relacionado con los factores productivos, tales como: tierras: hectáreas, recursos naturales, cantidad de demandantes, oferta de productos, diversificación, logística y distribución. Mientras que el segundo grupo corresponde a las relaciones de las empresas con el entorno, compuestas por: cantidad de oferentes, innovación, gestión empresarial, asociatividad, apoyo de instituciones, encadenamientos, certificaciones e infraestructura.

Por su parte, los actores sociales identificados fueron: productores individuales, asociaciones y cooperativas de Carrillo, empresas que exportan, cooperativas que exportan e instituciones participantes. Las relaciones esperadas se sintetizan en la siguiente figura. 


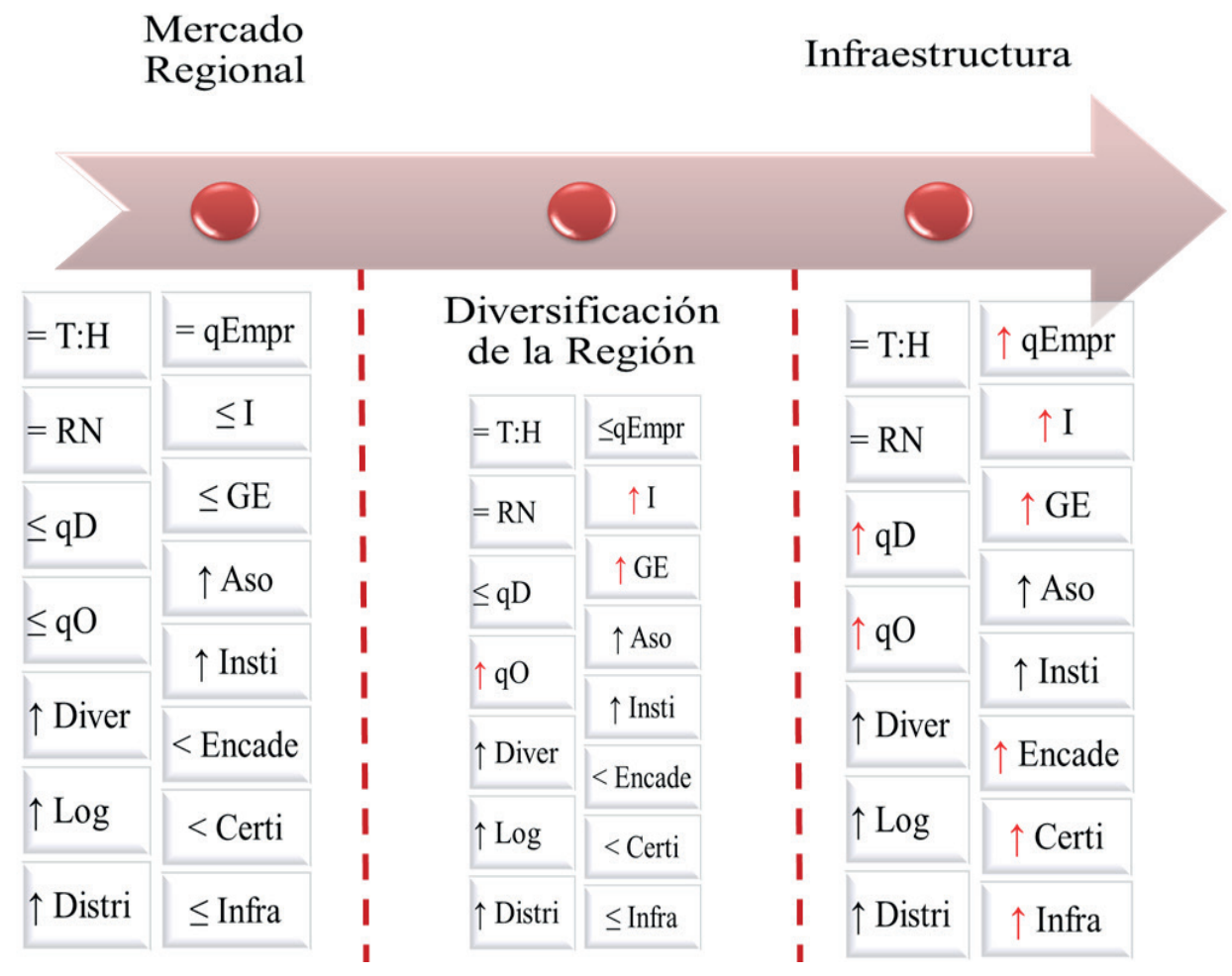

Figura 2. Escenarios proyectados

Fuente: Elaboración propia. Nota: Tierras: Hectáreas (T:H), Recursos Naturales (RN), Cantidad de demandantes (qD), Oferta de productos (qO), Diversificación (Diver), Logística (Log), Distribución (Distri), Cantidad de oferentes (qEmpr), Innovación (I), Gestión empresarial (GE), Asociatividad (Aso), Apoyo Instituciones (Insti), Encadenamientos (Encade), Certificaciones (Certi), Infraestructura (Infra), s permanece mayor o igual, $\uparrow$ Aumento Masivo, $<$ Aumento paulatino.

El comportamiento y relaciones de las variables por cada escenario se explican a continuación:

a. Mercado regional: Este es un proceso que se encuentra en curso y se puede considerar una de las primeras iniciativas que podrían generar la conformación de un STPA que incentive el crecimiento de este sector. El efecto en las variables se justifica de la siguiente manera:

- $=\mathrm{T}: \mathrm{H}$ : Se considera un factor fijo, en el sentido de que las extensiones de territorio de la provincia y el cantón son fijas. No se valora por los usos que se le den, sino por si estos aumentan o disminuyen. Lo mismo se proyecta para los demás escenarios proyectados.

- = RN: Los recursos naturales se consideran fijos, debido a que se busca incentivar la cultura ambiental en las empresas, si se generaliza el modelo de la adopción de 
certificaciones ambientales que poseen las empresas y cooperativas. Pese a que se requieren mayores recursos ante el aumento de producción, las empresas buscarán mitigar la tasa de extracción de una manera sustentable. En los tres escenarios esta variable permanece constante.

- SqD: La cantidad demandada de producto, comercializado a nivel local en el mercado, aumentaría con la conformación del mercado regional, ya que otros negocios, llámense sodas, hoteles, verdulerías, entre otros, se encuentran demandando productos y adquiriéndolos de otras zonas del país. Se indica que aumenta o permanece igual, debido a que puede que haya demandantes que no compren en el mercado y prefieran seguir comprando a los antiguos proveedores.

- SqO: La cantidad ofertada va aumentar debido a que hay gran cantidad de productores locales que se encuentran interesados en el proyecto y que indican que realizaría cambios en su oferta actual para comercializar la producción en el mercado regional.

- $\uparrow$ Diver: Estos oferentes que indican que aumentarían la producción también estarían interesados en diversificar la producción.

- $\uparrow$ Log: Esta aumentaría ya que se $\uparrow q \mathrm{O}$ y $\uparrow$ Diver. Nota: El proceso logístico inicia desde la compra de insumos, la preparación de la tierra para el cultivo, la cosecha y el transporte, hasta llegar al consumidor final.

- $\uparrow$ Distri: Esta aumentaría ya que se $\uparrow \mathrm{qO}$ y $\uparrow$ Diver y $\uparrow$ Log.

- = qEmpr: Para este escenario se considera que la cantidad de empresas permanece igual, las existentes buscarán vincularse con otras para generar $\uparrow q \mathrm{O}$ y $\uparrow$ Diver.

- $\leq \mathrm{I}$ : Las empresas incluirán $\uparrow q \mathrm{O}$ y $\uparrow$ Diver, por lo cual deben implementar un aumento en la innovación de los procesos y productos.

- SGE: La Gestión empresarial se modificará, la empresa debe reinventar sus metas y procesos ante la variación de $\uparrow q \mathrm{O}, \uparrow$ Diver, $\leq \mathrm{I}, \uparrow \log \mathrm{y} \uparrow$ Distri. Esta variable también involucra el personal o recurso humano que puede que aumente o se mantenga igual.

- $\uparrow$ Aso: Como $=\mathrm{qEmpr}$, entonces se aumentará la asociatividad o bien los join venture para poder participar en el mercado regional.

- $\uparrow$ Insti: debido al incentivo del mercado regional, las instituciones tendrán un papel de mayor participación: el INDER trabajará de cerca con asociaciones a las cuales dona hectáreas, el MAG dará seguimiento y capacitaciones a proyectos de agricultura responsable, PROCOMER identificará posibles empresas, asociaciones o cooperativas con características de exportador para capacitarlas.

- < Encade: Se generarán mayores encadenamientos al $\uparrow \log y \uparrow$ Distri.

- < Certi: Las empresas, asociaciones y cooperativas podrían adquirir certificaciones utilizadas a nivel local como Carbono Neutro, Esencial Costa Rica y la de Buenas 
Prácticas Agrícolas del MAG, con el fin de adoptar prácticas responsables y así mantener $=\mathrm{RN}$.

- SInfra: La infraestructura puede que aumente o permanezca igual. En el sentido de que para el mercado regional se construirá el edificio del PIMA, también se pretende la ampliación de la terminal de carga lo cual aumentaría la infraestructura, sin embargo, el acceso a las vías terrestres no ha mejorado, por lo cual los caminos permanecen igual ante nuevos portales para colocar producto.

b. Diversificación de la región: Este escenario se refiere a diversificar la producción de la región en su máxima amplitud del territorio. Es decir, al promover un STPA se dinamizaría el sector primario y, de la mano con el ya conformado mercado regional, se debería incentivar mercados cautivos en todo el territorio de la Región Chorotega, con el incentivo de la asociatividad y el impulso de estos pequeños agricultores a crecer. Dentro de este escenario, las variables $=\mathrm{T}: \mathrm{H},=\mathrm{RN}, \leq \mathrm{qD}, \uparrow$ Diver, $\uparrow$ Log, $\uparrow$ Distri, $\leq \mathrm{qEmpr}, \uparrow$ Aso, $\uparrow$ Insti, $<$ Encade, $<$ Certi e $\leq$ Infra presentan el mismo comportamiento del primer escenario, por tanto la misma razón de cambio. Entre las variables que cambian con respecto al escenario anterior, se encuentran:

- $\uparrow$ qO: Aumenta rotundamente la cantidad ofertada, las empresas deciden consumir la producción local.

- $\uparrow$ I: Las empresas han debido de aumentar por completo los procesos de innovación, deben realizar una constante innovación en la mejora de procesos para lograr mantener la producción que cubra la demanda interna del mercado regional.

- $\uparrow$ GE: Aumenta por completo ante una diversificación decisiva se deberá incorporar cambios en la estructura organizacional e incluir nuevo recurso humano, para cubrir con la producción que permite la participación en el mercado regional.

c. Infraestructura: Esta inicialmente se definió como la composición del sector financiero, la infraestructura vial y los servicios sociales básicos, sin embargo, para este escenario se realizará especial énfasis en la infraestructura vial, debido a que con el desarrollo de los pueblos, lo referente al sistema financiero y los servicios sociales básicos se supone van a mejorar.

De esta manera, se proyecta lo siguiente de las variables: $=\mathrm{T}: \mathrm{H},=\mathrm{RN}, \uparrow$ Diver, $\uparrow$ Log, $\uparrow$ Distri, $\uparrow \mathrm{I}, \uparrow \mathrm{GE}, \uparrow$ Aso, $\uparrow$ Insti, mientras que las que experimentan variaciones con respecto a los escenarios anteriores corresponden a:

- $\uparrow \mathbf{q O} \mathbf{y} \uparrow \mathbf{q E m p r}$ : Ante las mejoras de las condiciones del sector agrícola de la provincia, los productores de otras zonas se verán interesados en participar en el mercado regional y en producir en tierras de la Región Chorotega, aumentando así ambos componentes. 
Revista de Política Económica • EISSN: 2215-4167

Vol. 3 (1) • Enero- Junio, 2018: X-44-63

DOI: http://dx.doi.org/10.15359/peds.3-1.3

Porras Acevedo

URL: http://www.revistas.una.ac.cr/politicaeconomica

- $\uparrow$ qD: Esta aumentaría ante el aumento masivo de infraestructura, habrá posibilidades de colocar el producto no solo en el mercado regional sino en el exterior ante la mejora de vías más eficientes y de menor costo.

- $\uparrow$ Certi: Ante la posibilidad de comercializar en otros mercados, se plantea la necesidad de adquirir certificaciones según las exigencias del mercado a incursionar.

- $\uparrow$ Infra: Aumento masivo en infraestructura debido a la mejora en el camino de acceso a la ampliación terminal de carga y al mercado mayorista PIMA, la ampliación carretera internacional tramo Liberia-La Cruz, la creación del canal seco de Santa Cecilia-Limón y el Ferry Caldera-El Salvador. Estos corresponden a los posibles escenarios de mejora para la comercialización de bienes agroalimentarios, pues una mejora en infraestructura reduce el tiempo de transporte, lo cual, a su vez, se traslada a los costos. Por esto, estas mejoras incentivarían nuevas rutas a nivel internacional.

- $\uparrow$ Encade: Debido al aumento de todas las variables anteriores, se producirán mayores encadenamientos entre las empresas que demandarán más insumos y servicios para el cumplimiento de todo el proceso logístico de la empresa, asociación o cooperativa.

Para el logro de estos escenarios, se deben formular estrategias que permitan alcanzar el escenario desafío. No se realizará la elección de uno en específico, sino que se verá como un proceso para la mejora y el establecimiento de un STPA en la región, es decir, se tendrá un escenario tendencial o desafío.

\section{Conclusiones y recomendaciones de política}

Los sistemas territoriales se constituyen como un espacio geográfico que busca incentivar una actividad productiva específica. En este caso, se refiere a la producción agroalimentaria. Otros nombres vinculados y relacionados al concepto de sistema territorial son los distritos industriales, el clúster o los sistemas de innovación.

Se plantea el logro del desarrollo territorial de la provincia, mediante la articulación entre los actores, los cuales se empoderan y vuelven partícipes del desarrollo territorial de la localidad, sean estas empresas y gobiernos locales, los cuales integrados con el inventario de recursos disponibles y los recursos y activos específicos lograrán la mejor asociatividad para el desarrollo.

Como punto de partida, se requieren espacios que promuevan el sector agroalimentario, para aumentar su dinamismo y participación, y también que estos actores busquen la diversificación y, por consiguiente, aumente la oferta de productos. Esto se lograría con el incentivo el mercado regional, con partición y abastecimiento de productores locales.

El aumento de la producción y la diversificación de la producción experimentada por las empresas trae consigo la mejora en otros componentes como la productividad, la innovación, la gestión empresarial, la logística y la creación de nuevos empleos. 
Los gobiernos locales, por su parte, deben incentivar estos sectores con la mejora de la infraestructura, la cual cubre infraestructura vial, sistema financiero y servicios sociales básicos, así como la extensión de capacitación.

Los STPA promueven una alta asociatividad y encadenamientos. Además, incluyen el medio ambiental como una variable a circunscribir en los procesos, por lo cual se debe fomentar medidas como las certificaciones para promover las buenas prácticas. El incentivo de los STPA es que se constituyen como una alternativa que promueve la necesidad de garantizar la seguridad y la soberanía alimentaria de las regiones con el abastecimiento de un mercado mediante los productos locales, mejorando calidad de vida y disminuyendo la pobreza, el desempleo y la migración.

Se recomienda realizar tres recomendaciones para el logro de la efectividad de los STPA. La primera es la mejora de la competitividad económica. Esta se dirige a brindar capacitación y mejora en la estructura organizacional de las empresas y asociaciones de la región, con el fin de fortalecerlas y lograr que se proyecten en el corto plazo al mercado local, en el mediano plazo mercado regional y nacional, y en el largo plazo al exterior.

La segunda recomendación tiene que ver con la asociatividad y encadenamiento, los cuales busca promover la generación de asociatividad o acuerdos de colaboración que favorezcan la participación en el mercado regional.

El bienestar social y la sostenibilidad ambiental, es la tercera recomendación. Se deben crear programas de reconocimiento a las empresas por la labor que realizan mediante las certificaciones. Estos pueden ser capacitaciones, donaciones económicas y en especie a las comunidades cercanas de su operación.

Por último, realizar un ordenamiento territorial, que permita realizar una sectorización de la producción de la Región Chorotega, así como las características de producción que se requiere y las condiciones climáticas.

\section{Referencias bibliográficas}

Alburquerque, F. (2003). Teoría y Práctica del enfoque de desarrollo local. Disponible en http://www.ciieberazategui.com.ar/junio2013/noviembre2013/Falburquerque-Coquimbo Masregion.pdf

Alburquerque, F. D. (2006). Enfoque del Desarrollo Territorial. En Guía de aprendizaje sobre integración productiva y desarrollo económico territorial (pp. 5-40). Sevilla: Instituto de Desarrollo Regional, Fundación Universitaria. Universidad de Sevilla.

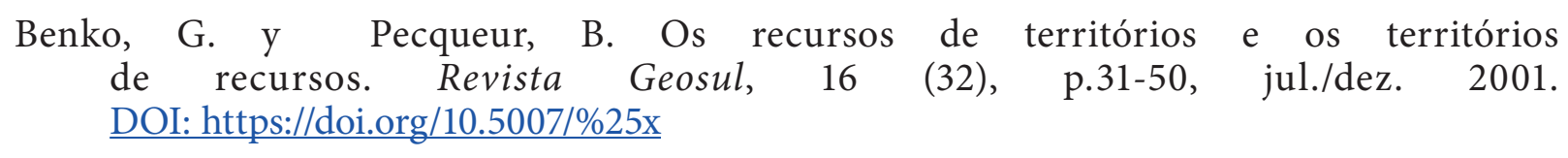


Colletis, G. \& B. Pecqueur (1995). "Dinámica territorial y factores de la competencia espacial". Vázquez Barquero, A. \& G. Garofoli, Desarrollo económico local en Europa. Madrid: Ed. Fundación Duques de Soria.

Comité Sectorial Agropecuario de la Región Chorotega (2007). Plan Regional de desarrollo, Sector Productivo Chorotega 2007-2010. Guanacaste, Costa Rica: Comité Sectorial Agropecuario de la Región Chorotega. Disponible en: http://www.mag.go.cr/bibliotecavirtual/E14-10830.pdf

Gallego, B. J. (2009). La articulación local-global de sistemas territoriales. Revista de estudios regionales (84), pp. 53-82.

Instituto Meteorológico Nacional (2015). El Niño del 2015 produce las condiciones climáticas más extremas en Costa Rica. Recuperado de http://gobierno.cr/el-nino-del-2015-producelas-condiciones-climaticas-mas-extremas-en-costa-rica/

Instituto Nacional de Estadística y Censos. (2014). Encuenta Nacional de Hogares, resultados generales. San Jose, Costa Rica: INEC.

Instituto Nacional de Estadística y Censos. (2015, Mayo). VI Censo Nacional Agropecuario, Informe general. Recuperado de http://www.mag.go.cr/bibliotecavirtual/a00338.pdf

Mendez, R. (2002). EURE SANTIAGO. Innovación y Desarrollo Territorial: Algunos debates Teoricos recientes. Recuperado de http://www.scielo.cl/scielo.php?pid=S0250$\underline{71612002008400004 \& \text { script }=\text { sci } \text { arttext }}$

Programa Integral de Mercado Agropecuario (PIMA) (2015). Mercado Regional Chorotega. San José: PIMA.

Secretaría Ejecutiva de Planificación Sectorial Agropecuaria (2014). Plan Sectorial de Desarrollo Agropecuario y Rural 2015-2018. SEPSA: Comité Sectorial Agropecuario de la Región Chorotega. Disponible en: http://www.mag.go.cr/bibliotecavirtual/E14-10830.pdf

\section{Anexos}

\section{Anexo 1. Entrevistas realizadas}

- Álvarez, I. (03 de Abril de 2016). Entrevista: PROCOMER. (S. Porras, Entrevistador)

- Bonilla, A. (2016, Abril 11). Entrevista Asociación de grupo de mujeres emprendedoras de Corralillos AGRUMEC. (S. Porras, Entrevistador)

- Calderón, M. (2016, Abril 17). Entrevista: Cámara Nacional de Agricultura y Agroindustria. (S. Porras, Entrevistador)

- Gutierrez, L. P. (2016, Abril 15). Entrevista Melones de la Península. (S. Porras, Entrevistador) 
Revista de Política Económica • EISSN: 2215-4167

Vol. 3 (1) • Enero- Junio, 2018: 44-63

DOI: http://dx.doi.org/10.15359/peds.3-1.3

Porras Acevedo

URL: http://www.revistas.una.ac.cr/politicaeconomica

- Mejicano, M. (2016, Abril 10). Entrevista Municipalidad de Carrillo. (S. Porras, Entrevistador)

- Mora, D. (2016, Abril 12). Entrevista JM Ivando. (S. Porras, Entrevistador )

- Pizarro, A. (2016, Abril). Entrevista Asentamiento la Hurraca. (S. Porras, Entrevistador)

- Quesada, R. Z. (13 de Abril de 2016). Entrevistas Programa Integral Mercados Agroalimentarios. (S. Porras, Entrevistador)

- Sánchez, O. (15 de abril de 2016). Entrevista Munisterio de Agricultura y Ganadería. (S. Porras, Entrevistador)

- Vásquez, K. (19 de Abril de 2016). Entrevistas Costeña S.A. (S. Porras, Entrevistador)

- Vásquez, O. (16 de Abril de 2016). Entrevista Ministerio de Agricultura y Ganadería. (S. Porras, Entrevistador)

- Venegas, R. (14 de Abril de 2016). Entrevista Melones del Sol. (S. Porras, Entrevistador)

- Zamora, J. L. (10 de Abril de 2016). Entrevista Exporpack S.A. (S. Porras, Entrevistador) 